\title{
99m Tc-cAbVCAM1-5 Imaging Is a Sensitive and Reproducible Tool for the Detection of Inflamed Atherosclerotic Lesions in Mice
}

\author{
Alexis Broisat*1,2, Jakub Toczek*1,2, Laurent S. Dumas ${ }^{1,2}$, Mitra Ahmadi ${ }^{1,2}$, Sandrine Bacot ${ }^{1,2}$, Pascale Perret ${ }^{1,2}$, \\ Lotfi Slimani $^{1,2}$, Gilles Barone-Rochette ${ }^{1-3}$, Audrey Soubies ${ }^{1,2}$, Nick Devoogdt ${ }^{4}$, Tony Lahoutte ${ }^{4,5}$, Daniel Fagret ${ }^{1,2}$, \\ Laurent M. Riou ${ }^{1,2}$, and Catherine Ghezzi ${ }^{1,2}$ \\ ${ }^{1}$ Unité 1039, INSERM, Grenoble, France; ${ }^{2}$ Radiopharmaceutiques Biocliniques, Université Joseph Fourier Grenoble 1, Grenoble, \\ France; ${ }^{3}$ Cardiology Department, Grenoble University Hospital, Grenoble, France; ${ }^{4}$ In vivo Cellular and Molecular Imaging \\ Laboratory, Vrije Universiteit Brussel (VUB), Brussels, Belgium; and ${ }^{5}$ Nuclear Medicine Department, UZ Brussel, Brussels, Belgium
}

\begin{abstract}
99mTc-cAbVCAM1-5, a single-domain antibody fragment directed against mouse or human vascular cell adhesion molecule 1 (VCAM1), recently has been proposed as a new imaging agent for the detection of inflamed atherosclerotic lesions. Indeed, in a mouse model of atherosclerosis, ${ }^{99 m T c-c A b V C A M 1-5}$ specifically bound to VCAM1-positive lesions, thereby allowing their identification on SPECT images. The purpose of the present study was to investigate ${ }^{99 m T c-}$ cAbVCAM1-5 imaging sensitivity using a reference statin therapy. Methods: Thirty apolipoprotein E-deficient mice were fed a westerntype diet. First, the relationship between the level of VCAM-1 expression and ${ }^{99 m}$ Tc-cAbVCAM1-5 uptake was evaluated in 18 mice using immunohistochemistry and autoradiography. Second, longitudinal SPECT/CT imaging was performed on control $(n=9)$ or atorvastatintreated mice $(0.01 \% \mathrm{w} / \mathrm{w}, n=9)$. Results: $99 \mathrm{mTc}$-cAbVCAM1-5 uptake in atherosclerotic lesions correlated with the level of VCAM-1 expression $(P<0.05)$. Atorvastatin exerted significant antiatherogenic effects, and 99mTc-cAbVCAM1-5 lesion uptake was significantly reduced in 35-wk-old atorvastatin-treated mice, as indicated by ex vivo $y$-well counting and autoradiography $(P<0.05)$. SPECT imaging quantification based on contrast-enhanced CT was reproducible (interexperimenter intraclass correlation coefficient, 0.97; intraexperimenter intraclass correlation coefficient, 0.90 ), and yielded results that were highly correlated with tracer biodistribution $(r=0.83 ; P<0.0001)$. Therefore, reduced ${ }^{99 m T c-C A b V C A M 1-5}$ uptake in atorvastatin-treated mice was successfully monitored noninvasively by SPECT/CT imaging $(0.87 \pm 0.06$ vs. $1.11 \pm 0.09$ percentage injected dose per cubic centimeter in control group, $P<0.05)$. Conclusion: ${ }^{99 m T c-}$ cAbVCAM1-5 imaging allowed the specific, sensitive, and reproducible quantification of VCAM-1 expression in mouse atherosclerotic

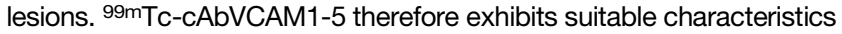
for the evaluation of novel antiatherogenic agents.
\end{abstract}

Key Words: 99mTc-cAbVCAM1-5; atherosclerosis; VCAM-1; molecular imaging

J Nucl Med 2014; 55:1678-1684

DOI: 10.2967/jnumed.114.143792

Received May 27, 2014; revision accepted Jul. 24, 2014.

For correspondence or reprints contact: Alexis Broisat, Laboratoire des Radiopharmaceutiques Bioclinique, INSERM 1039, Faculté de Médecine de Grenoble, Domaine de la Merci, 38700 La Tronche, France.

E-mail: alexis.broisat@inserm.fr

${ }^{*}$ Contributed equally to this work.

Published online Aug. 25, 2014.

COPYRIGHT (c) 2014 by the Society of Nuclear Medicine and Molecular Imaging, Inc.
A therosclerosis constitutes a worldwide health challenge (1). Despite major advances in imaging technologies (2), a major need for imaging probes targeting vulnerable plaque still exists. Such a tool could be used for diagnosis and prognosis as well as for the monitoring of treatment efficacy (3). A major criterion for the identification of vulnerable plaque is inflammation (4). Vascular cell adhesion molecule 1 (VCAM-1) plays a major role in the infiltration of inflammatory cells into atherosclerotic lesions (5). Indeed, in human atherosclerotic lesions, VCAM1 expression was observed at the level of the luminal endothelium as well as on neovessels, macrophages, and activated smooth muscle cells of advanced lesions (5-7). Therefore, the assessment of VCAM-1 expression levels in atherosclerotic plaques could potentially be highly relevant for identifying prone-to-rupture lesions (8). Because of the small size and location of vulnerable plaque, imaging agents with optimal characteristics are required. Single-domain antibody-derived radiotracers that originally were developed in the field of oncology (9) have recently been proposed by our group as a new class of imaging agent with optimal characteristics for cardiovascular imaging (10), and the radiolabeled anti-VCAM-1 camel antibody fragment ${ }^{99 \mathrm{~m}} \mathrm{Tc}-\mathrm{cAbVCAM} 1-5$ compound was selected among 10 VCAM-1-specific single-domain antibodies as the most potent candidate. Accordingly, aortic plaques were successfully visualized noninvasively by SPECT in an apolipoprotein E (ApoE)-deficient mouse model of atherosclerosis, with binding specificity being further demonstrated by in vivo competition experiments. Importantly, the production and selection processes of VCAM-1 single-domain antibody were specifically designed to produce cross-reactive murine and human binders. The nanomolar affinity of ${ }^{99 \mathrm{~m}} \mathrm{Tc}-\mathrm{cAbVCAM} 1-5$ for both human and murine VCAM-1 therefore makes the tracer potentially suitable for clinical translation (10).

The main objective of the present study was to investigate the sensitivity of ${ }^{99 \mathrm{~m}} \mathrm{Tc}-\mathrm{cAbVCAM} 1-5$ for the imaging of VCAM-1 expression in murine atherosclerotic lesions. High sensitivity is a key requirement for vulnerable plaque imaging and for preclinical assessment of the efficacy of novel antiatherogenic therapies. In accordance with the major role of inflammatory processes in the development of vulnerable plaque, the antiinflammatory effect of new therapeutic agents targeting the lipid profile, such as cholesterol esterase transfer protein inhibitors, is currently being investigated 
(11). In addition, therapeutic interventions aimed at directly targeting the inflammatory process are also under evaluation (3) using drugs such as methotrexate, canakinumab, or losmapimod (12-14). A specific and sensitive imaging agent targeting the inflammatory process in atherosclerotic lesions would therefore be of great relevance for the evaluation of novel antiatherogenic agents.

Statins are well established and widely used therapeutics in clinical practice, and atorvastatin has previously been used for the evaluation of new imaging agents in mouse models of atherosclerosis (15-17). In the present preclinical study, atorvastatin was used to evaluate the potential of ${ }^{99 \mathrm{~m} T c-c A b V C A M 1-5}$ for monitoring treatment efficacy in ApoE-deficient mice.

\section{MATERIALS AND METHODS}

\section{Animal Model}

All experimental procedures were in accordance with institutional guidelines and approved by the Animal Care and Use Committee (Comité d'Ethique) of the University of Grenoble.

Twelve 40-wk-old female ApoE-deficient mice (Charles River) fed a western-type diet were used for the ex vivo comparison between 99m Tc-cAbVCAM1-5 uptake in atherosclerotic lesions and VCAM-1 expression.

Eighteen female ApoE-deficient mice were used for noninvasive evaluation of a reference therapy. At the age of $15 \mathrm{wk}$, mice were fed either a western diet (control group, $n=9$ ) or a western diet supplemented with a reference therapy ( $0.01 \%$ atorvastatin) (treated group, $n=9)$ ). In these 2 groups, average food consumption and animal weight were assessed weekly and blood sampling was performed before and $10 \mathrm{wk}$ after the onset of the western diet for total cholesterol determination (BioMerieux). The effect of the statin therapy on ${ }^{99 m} \mathrm{Tc}-\mathrm{cAbVCAM} 1-5$ uptake in atherosclerotic lesions was evaluated in vivo using longitudinal SPECT/CT imaging on 25- and 35-wk-old mice and ex vivo by $\gamma$-well counting and autoradiography.

\section{Radiolabeling}

cAbVCAM1-5 labeling with ${ }^{99 \mathrm{~m}} \mathrm{Tc}$ was performed as previously described (10). Briefly, [ $\left.{ }^{99 \mathrm{~m}} \mathrm{Tc}\left(\mathrm{H}_{2} \mathrm{O}\right)_{3}(\mathrm{CO})_{3}\right]^{+}\left({ }^{99 \mathrm{~m}} \mathrm{Tc}\right.$-tricarbonyl) was synthesized by adding $1 \mathrm{~mL}$ of ${ }^{99} \mathrm{~m} \mathrm{TcO}_{4}{ }^{-}$solution to an Isolink kit (PSI). The vial was incubated at $100^{\circ} \mathrm{C}$ for $20 \mathrm{~min}$ and neutralized with $\mathrm{HCl}$. Then, $0.5 \mathrm{~mL}$ of ${ }^{99 \mathrm{~m}} \mathrm{Tc}$-tricarbonyl was added to $50 \mu \mathrm{g}$ of cAbVCAM1-5 and $50 \mu \mathrm{L}$ of $50 \mathrm{mM}$ carbonate buffer, $\mathrm{pH}$ 8.0, and this mixture was incubated for $90 \mathrm{~min}$ at $50^{\circ} \mathrm{C}$. The ${ }^{99 \mathrm{~m}} \mathrm{Tc}-\mathrm{cAbVCAM} 1-5$ solution was further gel-filtrated on Sephadex G25 (NAP-5; GE Healthcare) and filtered (0.22- $\mu \mathrm{m}$ Millex; Millipore). Radiochemical purity, determined by reverse-phase high-performance liquid chromatography, was more than $95 \%$ up to $6 \mathrm{~h}$ after radiolabeling.

\section{9mTc-cAbVCAM1-5 Uptake Versus VCAM-1 Expression}

99m Tc-cAbVCAM1-5 uptake in atherosclerotic lesions and VCAM-1 expression level were determined using autoradiographic imaging and immunohistochemistry, respectively $(n=12)$. Three hours after intravenous injection of $37 \pm 3 \mathrm{MBq}$ (mean $\pm \mathrm{SD}$ ), mice were euthanized using $\mathrm{CO}_{2}$. The aortic arch was cleaned in $10 \%$ formalin (Sigma) under a binocular tube (Discovery V8; Zeiss), embedded in optimal cutting temperature medium, and frozen in $-40^{\circ} \mathrm{C}$ isopentane. Serial $20-\mu \mathrm{m}$ - or 8 - $\mu \mathrm{m}$-thick adjacent slices were obtained for autoradiography and immunohistochemistry, respectively (HM $505 \mathrm{E}$; Microm). For the purpose of this study, a longitudinal orientation was preferred for it allowed simultaneous examination of several lesions, located in the ascending aorta; small curvature of the aortic arch; innominate, left common carotid, and left subclavian arteries; and descending aorta.

Autoradiography. Autoradiography was performed using overnight exposure with a phosphor imager (BAS-5000; Fujifilm). All slices were then stained using standard hematoxylin-eosin-safran trichrome staining (HES) and were digitalized, and HES and autoradiographic images were coregistered using GIMP2 and ImageJ software. Regions of interest were drawn on atherosclerotic lesions on the basis of HES staining. Internal references with known amounts of radioactivity were used to express tracer activity as percentage injected dose (\%ID) per gram after background and decay correction.

Immunohistochemistry. VCAM-1 immunostaining (sc-1504; Santa Cruz Biotechnology) was performed as previously described (10) on slices adjacent to those used for autoradiography and HES staining. The VCAM-1 expression level in atherosclerotic lesions $(n=94)$ was ranked as low, moderate, or strong by a trained observer masked to the autoradiographic images. This analysis was reproduced 2 mo later by the same observer to assess the reproducibility of the methodology.

\section{Noninvasive Evaluation of a Reference Therapy}

SPECT/CT Imaging. SPECT/CT acquisitions were performed $2 \mathrm{~h}$ after an intravenous injection of $55 \pm 5 \mathrm{MBq}$ (mean $\pm \mathrm{SD}$ ) of

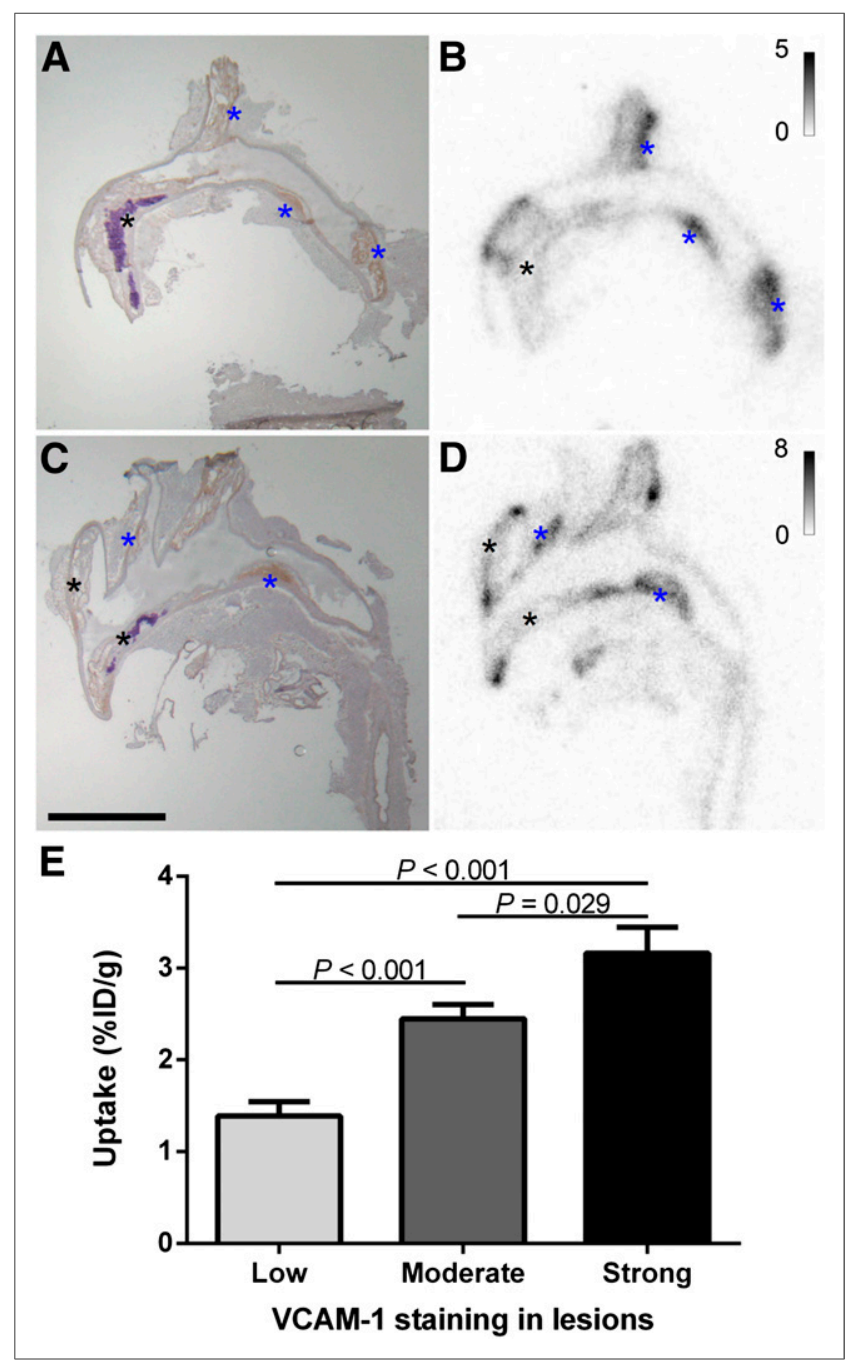

FIGURE 1. 99mTc-cAbVCAM1-5 uptake and VCAM-1 expression. (A and C) Representative VCAM-1 immunostaining performed on aortic arch lesions of ApoE-deficient mice. (B and D) 99mTc-cAbVCAM1-5 autoradiography imaging performed on adjacent slices. Strong 99mTc-CAbVCAM1-5 uptake was observed on atherosclerotic lesions expressing high level of VCAM-1 (blue asterisks), in comparison to atherosclerotic lesions expressing low levels of VCAM-1 (black asterisks). (E) This result was further confirmed by autoradiography quantification. Scale bar $=500 \mu \mathrm{m}$. 
TABLE 1

Effect of Treatment on Lesion Extent

\begin{tabular}{llll}
\hline \multicolumn{1}{c}{ Parameter } & Control & Treated & \% change \\
\hline Total cholesterol, chow diet $(\mathrm{mmol} / \mathrm{L})$ & $20.3 \pm 2.2$ & $18.8 \pm 2.2$ & $\mathrm{NS}$ \\
Total cholesterol, western diet $(\mathrm{mmol} / \mathrm{l})$ & $85.5 \pm 8.1$ & $60.7 \pm 6.6$ & $-29.1(P=0.034)$ \\
Lesion area $\left(\mathrm{mm}^{2}\right)$ & $1.07 \pm 0.11$ & $0.70 \pm 0.07$ & $-33.9(P=0.011)$ \\
\hline
\end{tabular}

99mTc-cAbVCAM1-5. The mice were anesthetized using 1.5\% isoflurane in a 1:1 mixture of room air and oxygen and then were placed in a temperature-controlled bed for SPECT/CT acquisitions focused on the thoracic region (nanoSPECT; Bioscan). First, the helicoidal SPECT acquisition was performed with 4 heads equipped with multipinhole collimators (nine 1.4-mm-diameter pinholes per head) using 24 projections and 45 min of acquisition. Then, a fast 1.5-min-long CT acquisition was performed immediately after retroorbital injection of $150 \mu \mathrm{L}$ of a vascular contrast agent (ioxaglate [Hexabrix]; Guerbet), using the following acquisition parameters: $65 \mathrm{kVp}, 180$ projections, and $500 \mathrm{~ms}$ per projection. Contrast-enhanced CT and SPECT acquisitions were reconstructed, fused, and quantified using dedicated software (InVivoScope; inviCRO, LLC).

SPECT/CT Quantification. SPECT quantification based on contrastenhanced CT was performed masked to the SPECT images. Briefly, 4 volumes of interest (VOIs) of $2.2 \mathrm{~mm}^{3}$ each were drawn at the level of the aortic sinus and ascending aorta on the basis of the enhanced CT images. ${ }^{99 \mathrm{~m}} \mathrm{Tc}$-cAbVCAM1-5 activity was expressed as $\% \mathrm{ID} / \mathrm{cm}^{3}$, and uptake in atherosclerotic lesions was defined as the maximum value from quantification of 4 VOIs. Moreover, to assess the inter- and intraobserver variability, 35-wk SPECT images were further quantified by 3 additional independent experimenters, and this quantification was reproduced 2 wk later by one of the experimenters.

Ex Vivo Quantification. After SPECT/CT imaging, anesthetized mice were euthanized using $\mathrm{CO}_{2}$, and ascending aortas, including the aortic sinus, were carefully harvested along with major organs. Tissue samples were weighed, and their activity was determined by $\gamma$-well counting (Wizard ${ }^{2}$; Perkin). The results were corrected for background and decay and expressed as $\% \mathrm{ID} / \mathrm{g}$. Adjacent transversal 40 - and $10-\mu \mathrm{m}$-thick cryosections were obtained at the level of the aortic sinus for autoradiographic imaging and immunostaining, respectively, and autoradiography quantification was performed after HES staining. Regions of interest were also used to determine lesion area in square millimeters. ${ }^{99 \mathrm{~m}} \mathrm{Tc}$-cAbVCAM1-5 uptake was expressed as \%ID per gram of lesion ("concentration") based on internal references. Alternatively, lesion volume in the analyzed slice was determined as the product between its surface and the slice thickness $(40 \mu \mathrm{m})$, and ${ }^{99 \mathrm{~m}}$ Tc-cAbVCAM1-5 uptake was then expressed as \%ID present in the analyzed slice ("total uptake").

\section{Statistical Analysis}

All results are presented as mean \pm SEM. $F$ testing was used for variance comparisons. The Student $t$, Mann-Whitney $U$, Pearson, intraclass correlation coefficient, and $\kappa$ tests were used for paired or unpaired datasets with equal variance, unpaired datasets with unequal variance, correlation analysis, and inter- or intraexperimenter variability, respectively. Differences were considered significant at a $P$ level of less than 0.05 .

\section{RESULTS}

\section{9mTc-cAbVCAM1-5 Uptake Versus VCAM-1 Expression}

Heterogeneous VCAM-1 expression levels were observed after immunostaining of longitudinal slices from the aortic arch of ApoEdeficient mice (Figs. 1A and 1C). VCAM-1 was expressed by endothelial as well as intraplaque cells. Intraobserver ranking of VCAM-1 expression level was found to be reproducible $(\kappa=$ 0.70). Robust ${ }^{99 \mathrm{~m} T c-c A b V C A M 1-5}$ uptake was observed after autoradiographic imaging of VCAM-1-positive lesions (Figs. 1B and 1D). Autoradiography quantification confirmed the relationship between 99mTc-cAbVCAM1-5 uptake and the level of VCAM-1 expression (Fig. 1E). A graded tracer uptake of 1.4 $\pm 0.2,2.5 \pm 0.2$, and $3.2 \pm 0.3 \% \mathrm{ID} / \mathrm{g}$ was observed in low, moderate, and strong VCAM-1-expressing lesions, respectively $(P<0.001$ between low and moderate or strong, and $P=0.029$ between moderate and high).

\section{Noninvasive Evaluation of a Reference Therapy}

Lesion Extent. The results are presented in Table 1 and Supplemental Figure 1. Similar cholesterol levels were observed between treated and control animals before they were switched from chow to a western diet containing atorvastatin (treated) or not (control) $(20.3 \pm 2.2$ vs. $18.8 \pm 2.2 \mathrm{mmol} / \mathrm{L}, P=$ not statistically significant [NS]). Average atorvastatin intake was $12.9 \pm 0.2 \mathrm{mg} / \mathrm{kg} / \mathrm{d} / \mathrm{animal}$

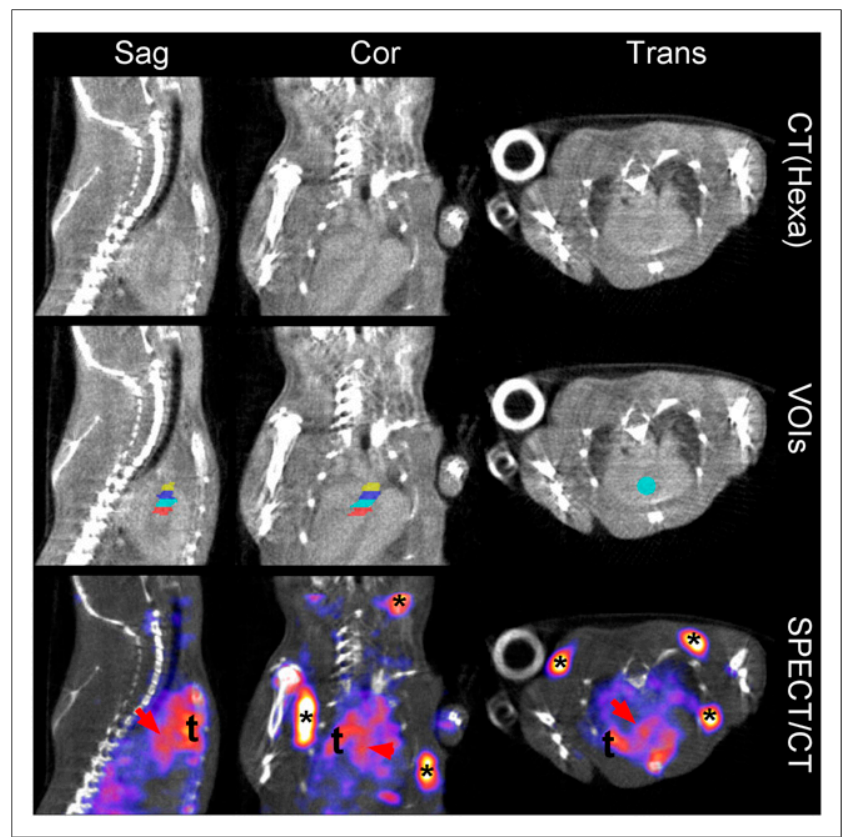

FIGURE 2. In vivo SPECT/CT imaging. Representative example of sagittal (first column), coronal (second column) and transaxial (third column) views centered on ascending aorta of control group mouse are presented here. Myocardial cavities and major vessels including aorta were easily identifiable on Hexabrix-enhanced CT images (first row), allowing precise definition of $4 \mathrm{VOls}$ along aortic sinus and ascending

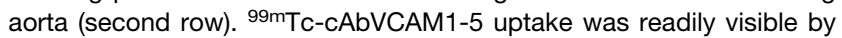
SPECT within aorta (red arrow), as well as in tissues constitutively expressing VCAM-1 such as lymph nodes (asterisks) and thymus (t). SPECT scale was normalized from 0.3 to $3 \% \mathrm{ID} / \mathrm{cm}^{3}$. 


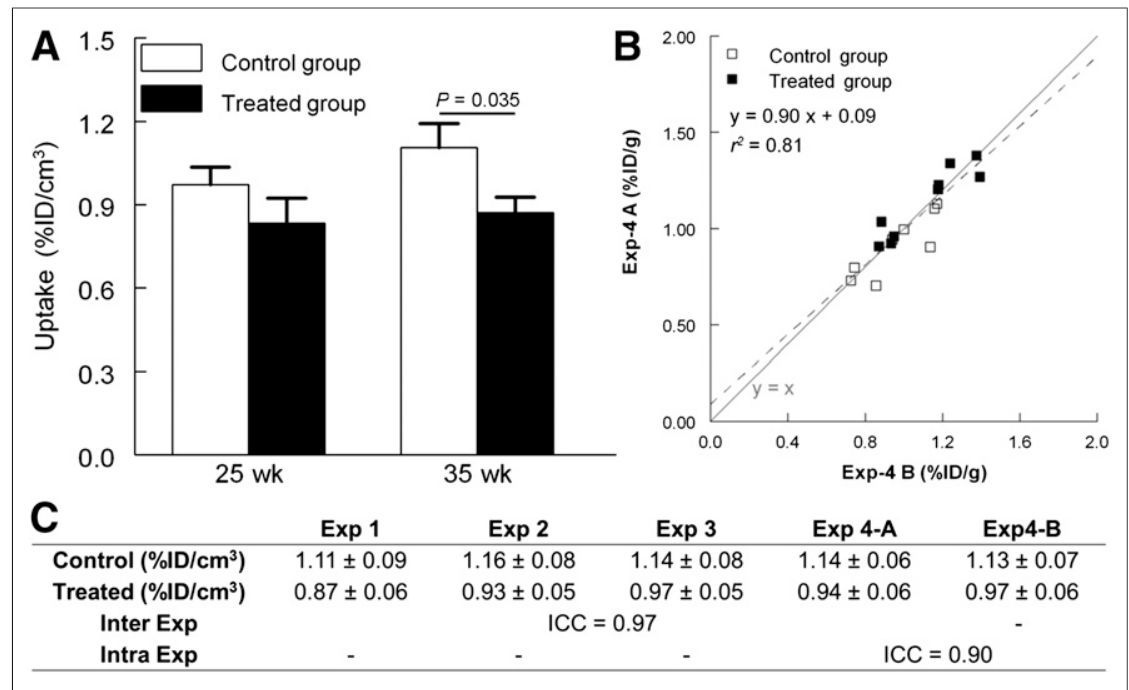

FIGURE 3. SPECT/CT quantification. Reduction of 99mTc-cAbVCAM1-5 uptake in treated mouse aorta was successfully monitored in vivo by SPECT/CT imaging (A). Furthermore, SPECT analysis based on contrast-enhanced CT provided robust quantifications, as determined by intraexperimenter correlation (B) and inter- and intraexperimenter intraclass correlation coefficient (ICC) (C).

and resulted in a significant $29.1 \%$ decrease in total cholesterol level in the atorvastatin-treated group in comparison with the control group $(60.7 \pm 6.6$ vs. $85.5 \pm 8.1 \mathrm{mmol} / \mathrm{L} ; P=0.034)$. Lesion extent in the aortic sinus was significantly reduced by $39.9 \%$ in the treated group in comparison to that in the control group $\left(0.70 \pm 0.07\right.$ vs. $\left.1.07 \pm 0.11 \mathrm{~mm}^{2}, P=0.011\right)$ (Table 1 ; Supplemental Fig. 1 [supplemental materials are available at http:// jnm.snmjournals]). Finally, modest variations in VCAM-1 expression in the aortic sinus were observed between control and treated animals after immunohistochemistry (Supplemental Fig. 1).

In Vivo SPECT/CT Imaging. ${ }^{99 \mathrm{~m} T c-c A b V C A M 1-5}$ allowed the noninvasive monitoring of atorvastatin effect on atherosclerosis. 99m Tc-cAbVCAM1-5 uptake in aortic lesions was readily visible on SPECT images (Fig. 2), and the trend toward a reduction of 99mTc-cAbVCAM1-5 uptake that was observed in 25-wk-old atorvastatin-treated mice $\left(0.83 \pm 0.09 \% \mathrm{ID} / \mathrm{cm}^{3}\right.$ vs. $0.97 \pm 0.06$ $\% \mathrm{ID} / \mathrm{cm}^{3}$ in the control group, $P=\mathrm{NS}$ ) reached statistical significance at $35 \mathrm{wk}\left(0.87 \pm 0.06 \% \mathrm{ID} / \mathrm{cm}^{3}\right.$ vs. $1.11 \pm 0.09 \% \mathrm{ID} / \mathrm{cm}^{3}$ in the control group, $P=0.035)$. Enhanced-CT acquisitions allowed accurate delineation of the ascending aorta in all animals, and enhanced-CT-based SPECT quantification was reproducible, with inter- and intraexperimenter intraclass correlation coefficients of 0.97 and 0.90, respectively (Fig. 3).

Ex Vivo $\gamma$-Well Counting. ${ }^{99 \mathrm{~m}} \mathrm{Tc}-\mathrm{cAbVCAM} 1-5$ biodistribution is presented in Table 2. ${ }^{99 \mathrm{~m}} \mathrm{Tc}-\mathrm{cAbVCAM} 1-5$ uptake in atorvastatintreated animals was significantly decreased by $24.1 \%$ in the ascending aorta of atorvastatin-treated animals $(0.9 \pm 0.1$ vs. $1.2 \pm 0.1$ $\% \mathrm{ID} / \mathrm{g}, P=0.015)$. The tracer was eliminated mainly through the kidneys $(138.7 \pm 8.6 \% \mathrm{ID} / \mathrm{g})$, with minimal uptake in all other investigated tissues $(<2 \% \mathrm{ID} / \mathrm{g})$ except the lymphoid organs. Indeed, tracer uptake in the spleen, thymus, bone marrow, and lymph node was $8.8 \pm 0.7,2.1 \pm 0.2,4.0 \pm 0.4$, and $2.8 \pm 0.3 \% \mathrm{ID} / \mathrm{g}$, respectively.

Ex Vivo Autoradiography. In both groups, robust 99m Tc-cAbVCAM1-5 uptake in atherosclerotic lesions was readily observable at the level of the aortic sinus (Supplemental Figs. 1A-1D). A strong and statistically significant $41.5 \%$ decrease in ${ }^{99 m}$ Tc-cAbVCAM1 total uptake was observed in the atorvastatin-treated group in comparison to that in the control group (Table 3). ${ }^{99 \mathrm{~m}} \mathrm{Tc}-\mathrm{cAbVCAM} 1$ uptake represented $8.9 \times 10^{-5} \pm 1.1 \times 10^{-5} \%$ ID per slice in the control group and $5.210^{-5} \pm 0.910^{-5} \% \mathrm{ID}$ per slice in the treated group. Interestingly, autoradiography quantification indicated a trend toward a $15.2 \%$ decrease of 99m Tc-cAbVCAM1-5 concentration in atherosclerotic lesions from atorvastatintreated animals when tracer uptake was corrected for plaque area $(1.8 \pm 0.2$ $\% \mathrm{ID} / \mathrm{g}$ vs. $2.1 \pm 0.2 \% \mathrm{ID} / \mathrm{g}$ in the control group, $P=\mathrm{NS}$ ),

Comparison Between SPECT/CT, Autoradiography, and $\gamma$-Well Counting Data. Further data analysis indicated that SPECT/CT quantification was strongly correlated to ex vivo $\gamma$-well counting biodistribution $(r=0.83 ; P<$ 0.0001) (Fig. 4A). Moreover, the results from SPECT/CT image quantification correlated significantly with total 99m Tc-cAbVCAM1 uptake in aortic sinus lesions as determined by autoradiography $(r=0.51 ; P=0.04)$ rather than to ${ }^{99 \mathrm{~m} T c-c A b V C A M} 1$ concentration or to lesion area $(P=\mathrm{NS}$ for both) (Figs. 4B-4D).

\section{DISCUSSION}

Atorvastatin significantly reduced the extent of lesions in the aortic sinus of ApoE-deficient mice, and this effect was successfully monitored noninvasively using ${ }^{99 \mathrm{~m} T c-c A b V C A M 1-5}$ molecular

TABLE 2

Ex Vivo Biodistribution of 99mTc- cAbVCAM1-5 3 Hours After Injection in ApoE-Deficient Mice

\begin{tabular}{lrr}
\hline \multicolumn{1}{c}{ Site } & \multicolumn{1}{c}{ Control } & \multicolumn{1}{c}{ Treated } \\
\hline Ascending aorta & $1.2 \pm 0.1$ & $0.9 \pm 0.1^{\star}$ \\
\hline Blood & $0.6 \pm 0.1$ & $0.5 \pm 0.0$ \\
\hline Heart & $0.3 \pm 0.0$ & $0.3 \pm 0.0$ \\
\hline Lung & $1.7 \pm 0.1$ & $1.8 \pm 0.1$ \\
\hline Liver & $1.5 \pm 0.1$ & $1.4 \pm 0.1$ \\
\hline Skeletal muscle & $0.1 \pm 0.0$ & $0.3 \pm 0.1$ \\
\hline Salivary gland & $0.7 \pm 0.2$ & $0.7 \pm 0.1$ \\
\hline Thyroid & $1.2 \pm 0.2$ & $0.9 \pm 0.2$ \\
\hline Stomach & $0.7 \pm 0.1$ & $0.7 \pm 0.1$ \\
\hline Bile & $0.4 \pm 0.0$ & $0.5 \pm 0.1$ \\
\hline Kidney & $138.7 \pm 8.6$ & $165.0 \pm 6.8$ \\
\hline Spleen & $8.8 \pm 0.7$ & $7.9 \pm 0.5$ \\
\hline Thymus & $2.1 \pm 0.2$ & $1.8 \pm 0.2$ \\
\hline Bone marrow & $4.0 \pm 0.4$ & $4.1 \pm 0.7$ \\
\hline Lymph note & $2.8 \pm 0.3$ & $2.7 \pm 0.2$ \\
\hline${ }^{*} P=0.015$ vs. control group. & \\
Data are \%ID/g (mean $\pm S E M)$. & \\
\hline
\end{tabular}


TABLE 3

Ex Vivo Autoradiography Quantification on Aortic Sinus Lesions

\begin{tabular}{llllc}
\hline \multicolumn{1}{c}{ Parameter } & \multicolumn{1}{c}{ Unit } & Control & Treated & \% change \\
\hline Total uptake & $\%$ ID/slice $\left(0.10^{-6}\right)$ & $8.9 \pm 1.2$ & $5.2 \pm 0.9$ & $-41.5(P=0.024)$ \\
Concentration & $\% \mathrm{ID} / \mathrm{g}$ & $2.1 \pm 0.2$ & $1.8 \pm 0.2$ & $\mathrm{NS}$ \\
\hline
\end{tabular}

imaging with high reproducibility. ${ }^{99 \mathrm{~m}} \mathrm{Tc}-\mathrm{cAbVCAM} 1-5$ is therefore a specific, sensitive, and reproducible tool for the imaging of VCAM-1 expression in murine atherosclerotic lesions.

\section{Effect of Statin Therapy}

The antiatherosclerotic effects of statins are mediated mainly by their cholesterol-lowering properties, leading to reduced plaque volume (18). In addition, statins also exert pleiotropic effects that go beyond the drop in plasma cholesterol and result mainly in an inhibition of inflammatory processes responsible for vulnerable plaque development $(19,20)$. However, depending on the dose and duration of the treatment and on study design, the reduction in plasma cholesterol, plaque volume, and inflammatory markers is not always consistently or concomitantly observed in experimental studies. Indeed, in an ApoE-deficient mouse model of carotid artery lesions induced by perivascular collar placement, Bot et al. observed that a low dose of atorvastatin $(0.003 \% \mathrm{w} / \mathrm{w})$ significantly reduced lesion size by $40 \%$ despite no reduction in total plasma cholesterol or macrophage-positive areas (15). Conversely, a 12-wk atorvastatin treatment $(1 \mathrm{mg} / \mathrm{kg} / \mathrm{d})$ resulted in a significant reduction in the circulating inflammatory markers interleukin-6 and monocyte chemoattractant protein-1 as well as macrophage infiltration in aortic lesions of apoE-deficient mice despite no significant change in plasma cholesterol and plaque area (21).

The apoE-deficient mice fed a western diet in the present study had a mean total blood cholesterol level of $85.5 \pm 8.1 \mathrm{mmol} / \mathrm{L}$, in good accordance with the $28-114 \mathrm{mmol} / \mathrm{L}$ range reported for a similar model by Nakashima et al. (22). Supplementation of the western diet with $0.01 \% \mathrm{w} / \mathrm{w}$ atorvastatin resulted in an expected administered dose of about $10 \mathrm{mg} / \mathrm{kg} / \mathrm{d}$ (16). Atorvastatin significantly decreased total cholesterol by $29 \%$ and lesion size by $40 \%$. These results are in accordance with the range of atorvastatin-induced modifications reported by other groups $(16,17)$. Modest changes in the lesional expression of VCAM-1 were observed in atorvastatin-treated animals, in contrast to other studies showing a significant $30 \%-80 \%$ reduction in VCAM-1 expression $(16,17,23)$. However, Nachtigal et al. (24) found no significant change in VCAM-1 lesion expression in double-knock-out low-density lipoprotein receptor-deficient ApoEdeficient mice in the presence of atorvastatin at a dose similar to that used in the present study.

Overall, atorvastatin administered at a dose of $0.01 \% \mathrm{w} / \mathrm{w}$ for 20 wk to ApoE-deficient mice fed a western diet therefore had significant antiatherogenic effects that allowed evaluation of the potential of ${ }^{99 \mathrm{~m}} \mathrm{Tc}-\mathrm{cAbVCAM} 1-5$ for treatment effect monitoring.

\section{Sensitivity of ${ }^{99 m}$ Tc-cAbVCAM1-5 Imaging}

99mTc-cAbVCAM1-5 uptake occurred in atherosclerotic lesions and in lymphoid organs constitutively expressing VCAM-1 after tracer injection to ApoE-deficient mice fed a western diet, in accordance with previously published results (10). Autoradiography and immunohistochemistry indicated that ${ }^{99 \mathrm{~m} T c-c A b V C A M 1-5}$ uptake in atherosclerotic lesions significantly increased together with the level of VCAM-1 expression. Importantly, ${ }^{99 m}$ Tc-cAbVCAM1-5 lesion uptake was significantly reduced in 35-wk-old atorvastatintreated mice as indicated by ex vivo $\gamma$-well counting and autoradiography as well as in vivo noninvasive SPECT/CT molecular imaging. In vivo image quantification was reproducible and yielded results that correlated strongly with tracer biodistribution as determined ex vivo.

More precisely, VOIs from in vivo images were reproducibly drawn at the level of the aortic sinus on the basis of enhanced-CT images. In the hypothesis that the VOI exclusively contained atherosclerotic lesional tissues and according to the fact that 99m Tc-cAbVCAM1-5 uptake in murine atherosclerotic lesion significantly increased together with the level of VCAM-1 expression, SPECT quantification would then have directly reflected the level of VCAM-1 expression in atherosclerotic lesions. However, because of the relatively small size of atherosclerotic lesions, and despite the use of a state-of-the-art, submillimeter-resolution system, partial-volume effect necessarily occurred during SPECT imaging of murine aortic plaques. Therefore, the VOI used for the assessment of tracer uptake in atherosclerotic lesions contained a mixture of the various tissues composing the aortic sinus-that
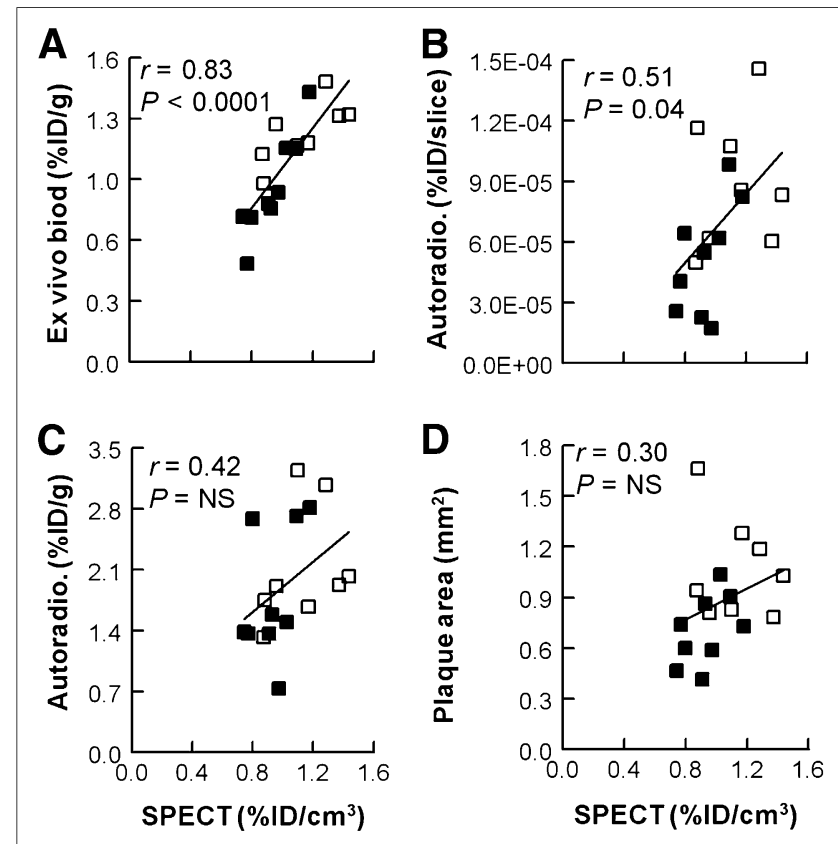

FIGURE 4. Comparison between in vivo and ex vivo quantifications of 99mTc-cAbVCAM1-5 uptake in atherosclerotic lesions. SPECT/CT quantification was compared with ex vivo biodistribution in samples consisting of aortic sinus and ascending aorta (A), as well as with autoradiography quantification results expressed either as total uptake in lesions per slice (B) or as concentration (C), and with plaque area (D). White markers $=$ control group; black markers $=$ treated group. 
is, atherosclerotic lesions, normal vessel wall, and blood-rather than the atherosclerotic lesion only. Consequently, the total activity in a VOI, and therefore the result of in vivo quantification, depended not only on the level of VCAM-1 expression in the lesions but also on the volume of lesions included in the VOI, and therefore lesion extent. This was demonstrated in the present study by the fact that the results from SPECT/CT image quantification correlated significantly with $99 \mathrm{~m}$ Tc-cAbVCAM1 total uptake in aortic sinus lesions as defined by the product of 99m Tc-cAbVCAM1 concentration in lesion-by-lesion volume, rather than with either of these 2 parameters considered separately. In conclusion, ${ }^{99 m} \mathrm{Tc}-\mathrm{cAbVCAM} 1-5$ SPECT/CT imaging allowed the sensitive and reproducible noninvasive quantification of the net effect of a reference statin therapy on lesion size and inflammation in the apoE-deficient mouse model of atherosclerosis.

\section{Comparison with Other Radiotracers and Molecular Targets}

The sensitivity of $99 \mathrm{~m}$ Tc-cAbVCAM1-5 imaging favorably compared with previously evaluated radiotracers. In a rabbit model of atherosclerosis, Haider et al. observed a decrease in the uptake of 2 SPECT agents directed against matrix metalloproteinases and apoptosis (annexin-A5) after treatment with fluvastatin (25). Although SPECT image quantification was not performed, these qualitative visual observations were further confirmed by quantitative ex vivo analysis. Similarly, a significant $30 \%$ reduction in ${ }^{18} \mathrm{~F}-4 \mathrm{~V}$ was found by in vivo PET image quantification in a model similar to that used in the present study (23). Several studies have investigated the potential of ${ }^{18} \mathrm{~F}$-FDG for the noninvasive assessment of treatment efficacy. Such potential has been validated in rabbits using the antioxidant probucol (26), thereby allowing the use of ${ }^{18} \mathrm{~F}$-FDG for the evaluation of novel therapeutics in preclinical studies $(27,28)$ and clinical studies $(12,29)$. However, high myocardial uptake remains a potential limitation for the imaging of coronary lesions with ${ }^{18} \mathrm{~F}-\mathrm{FDG}$ despite the use of imaging protocols aimed at reducing myocardial tracer uptake (30).

VCAM-1 protein is strongly and specifically expressed during the inflammatory process and therefore constitutes a highly relevant target for vulnerable plaque imaging, similarly to other inflammatory markers such as macrophages. Other biologic processes related to plaque rupture such as apoptosis, matrix degradation, or neoangiogenesis (31) have been proposed as potential targets. These processes occur at different times and intraplaque locations over the course of plaque development. Ultimately, prospective clinical trials will be needed to select the most appropriate target and corresponding imaging agent.

\section{CONCLUSION}

SdAbs-derived radiotracers were initially developed in the field of oncology, and a first phase I clinical trial is currently being performed with an anti-human epidermal growth factor receptor 2 radiotracer for PET imaging of breast cancer patients. AntiVCAM-1 single-domain antibody has extended the field of singledomain antibody imaging to cardiovascular applications. As demonstrated in the present study, ${ }^{99 \mathrm{~m} T c-c A b V C A M 1-5}$ is a sensitive tool for the noninvasive imaging of atherosclerosis. Indeed, ${ }^{99 \mathrm{~m}} \mathrm{Tc}$-cAbVCAM1-5 uptake in mouse atherosclerotic lesions correlated with the level of VCAM-1 expression, and the effect of a reference therapy was successfully and reproducibly monitored noninvasively by SPECT/CT imaging quantification. 99m Tc-cAbVCAM1-5 therefore exhibits suitable characteristics for evaluation of novel antiatherogenic agents.

\section{DISCLOSURE}

The costs of publication of this article were defrayed in part by the payment of page charges. Therefore, and solely to indicate this fact, this article is hereby marked "advertisement" in accordance with 18 USC section 1734 . This work was partly funded by the French program Investissement d'Avenir run by the Agence Nationale pour la Recherche, grant ANR-11-INBS-0006 (Infrastructure d'avenir en Biologie Santé), and grant ANR-09-TECS-017-01 (PLAQUIMAG). No other potential conflict of interest relevant to this article was reported.

\section{ACKNOWLEDGMENTS}

Antony Coradin, Pierre-Francois Meyer, and Audrey Genoud, students of Grenoble University, were involved in data collection and analysis during their training course in the Radiopharmaceutiques Biocliniques Laboratory.

\section{REFERENCES}

1. Sanz J, Moreno PR, Fuster V. The year in atherothrombosis. J Am Coll Cardiol. 2013;62:1131-1143.

2. Libby P, DiCarli M, Weissleder R. The vascular biology of atherosclerosis and imaging targets. J Nucl Med. 2010;51(suppl 1):33S-37S.

3. Berman JP, Farkouh ME, Rosenson RS. Emerging anti-inflammatory drugs for atherosclerosis. Expert Opin Emerg Drugs. 2013;18:193-205.

4. Fuster V, Moreno PR, Fayad ZA, Corti R, Badimon JJ. Atherothrombosis and high-risk plaque: part I: evolving concepts. J Am Coll Cardiol. 2005;46: 937-954.

5. O'Brien KD, McDonald TO, Chait A, Allen MD, Alpers CE. Neovascular expression of E-selectin, intercellular adhesion molecule-1, and vascular cell adhesion molecule-1 in human atherosclerosis and their relation to intimal leukocyte content. Circulation. 1996;93:672-682.

6. O'Brien KD, Allen MD, McDonald TO, et al. Vascular cell adhesion molecule-1 is expressed in human coronary atherosclerotic plaques: implications for the mode of progression of advanced coronary atherosclerosis. J Clin Invest. 1993; 92:945-951.

7. Davies MJ, Gordon JL, Gearing AJ, et al. The expression of the adhesion molecules ICAM-1, VCAM-1, PECAM, and E-selectin in human atherosclerosis. J Pathol. 1993;171:223-229.

8. Naghavi M, Libby P, Falk E, et al. From vulnerable plaque to vulnerable patient: a call for new definitions and risk assessment strategies: part II. Circulation. 2003;108:1772-1778.

9. Xavier C, Vaneycken I, D'huyvetter M, et al. Synthesis, preclinical validation, dosimetry, and toxicity of ${ }^{68} \mathrm{Ga}$-NOTA-anti-HER2 Nanobodies for iPET imaging of HER2 receptor expression in cancer. J Nucl Med. 2013;54:776-784.

10. Broisat A, Hernot S, Toczek J, et al. Nanobodies targeting mouse/human VCAM1 for the nuclear imaging of atherosclerotic lesions. Circ Res. 2012; 110:927-937.

11. Barter PJ, Rye K-A. Cholesteryl ester transfer protein inhibition as a strategy to reduce cardiovascular risk. J Lipid Res. 2012;53:1755-1766.

12. Elkhawad M, Rudd JHF, Sarov-Blat L, et al. Effects of p38 mitogen-activated protein kinase inhibition on vascular and systemic inflammation in patients with atherosclerosis. JACC Cardiovasc Imaging. 2012;5:911-922.

13. Everett BM, Pradhan AD, Solomon DH, et al. Rationale and design of the Cardiovascular Inflammation Reduction Trial: a test of the inflammatory hypothesis of atherothrombosis. Am Heart J. 2013;166:199-207.

14. Ridker PM, Thuren T, Zalewski A, Libby P. Interleukin-1 $\beta$ inhibition and the prevention of recurrent cardiovascular events: rationale and design of the Canakinumab Anti-inflammatory Thrombosis Outcomes Study (CANTOS). Am Heart J. 2011;162:597-605.

15. Bot I, Jukema JW, Lankhuizen IM, van Berkel TJC, Biessen EAL. Atorvastatin inhibits plaque development and adventitial neovascularization in ApoE deficient mice independent of plasma cholesterol levels. Atherosclerosis. 2011; 214:295-300.

16. Khanicheh E, Mitterhuber M, Xu L, Haeuselmann SP, Kuster GM, Kaufmann BA. Noninvasive ultrasound molecular imaging of the effect of statins on endothelial inflammatory phenotype in early atherosclerosis. PLOS ONE. 2013;8:e58761. 
17. Nahrendorf M, Jaffer FA, Kelly KA, et al. Noninvasive vascular cell adhesion molecule-1 imaging identifies inflammatory activation of cells in atherosclerosis. Circulation. 2006;114:1504-1511.

18. Brautbar A, Ballantyne CM. Pharmacological strategies for lowering LDL cholesterol: statins and beyond. Nat Rev Cardiol. 2011;8:253-265.

19. Rasmussen LM, Hansen PR, Nabipour MT, Olesen P, Kristiansen MT, Ledet T. Diverse effects of inhibition of 3-hydroxy-3-methylglutaryl-CoA reductase on the expression of VCAM-1 and E-selectin in endothelial cells. Biochem J. 2001; 360:363-370.

20. Ridker PM, Danielson E, Fonseca FAH, et al. Rosuvastatin to prevent vascular events in men and women with elevated C-reactive protein. $N$ Engl J Med. 2008;359:2195-2207.

21. Grothusen C, Bley S, Selle T, et al. Combined effects of HMG-CoA-reductase inhibition and renin-angiotensin system blockade on experimental atherosclerosis. Atherosclerosis. 2005;182:57-69.

22. Nakashima Y, Plump AS, Raines EW, Breslow JL, Ross R. ApoE-deficient mice develop lesions of all phases of atherosclerosis throughout the arterial tree. Arterioscler Thromb. 1994;14:133-140.

23. Nahrendorf M, Keliher E, Panizzi P, et al. ${ }^{18} \mathrm{~F}-4 \mathrm{~V}$ for PET-CT imaging of VCAM-1 expression in atherosclerosis. JACC Cardiovasc Imaging. 2009;2:1213-1222.

24. Nachtigal P, Pospisilova N, Jamborova G, et al. Atorvastatin has hypolipidemic and anti-inflammatory effects in apoE/LDL receptor-double-knockout mice. Life Sci. 2008;82:708-717.
25. Haider N, Hartung D, Fujimoto S, et al. Dual molecular imaging for targeting metalloproteinase activity and apoptosis in atherosclerosis: molecular imaging facilitates understanding of pathogenesis. J Nucl Cardiol. 2009; 16:753-762.

26. Ogawa M, Magata Y, Kato T, et al. Application of ${ }^{18}$ F-FDG PET for monitoring the therapeutic effect of antiinflammatory drugs on stabilization of vulnerable atherosclerotic plaques. J Nucl Med. 2006;47:1845-1850.

27. Vucic E, Dickson SD, Calcagno $\mathrm{C}$, et al. Pioglitazone modulates vascular inflammation in atherosclerotic rabbits monitored non-invasively with ${ }^{18} \mathrm{~F}$-fluorodeoxyglucose-PET/CT and black blood dynamic contrast enhancedMRI. JACC Cardiovasc Imaging. 2011;4:1100-1109.

28. Vucic E, Calcagno C, Dickson SD, et al. Regression of inflammation in atherosclerosis by the LXR agonist R211945: a noninvasive assessment and comparison with atorvastatin. JACC Cardiovasc Imaging. 2012;5:819-828.

29. Tawakol A, Fayad ZA, Mogg R, et al. Intensification of statin therapy results in a rapid reduction in atherosclerotic inflammation: results of a multicenter fluorodeoxyglucose-positron emission tomography/computed tomography feasibility study. J Am Coll Cardiol. 2013;62:909-917.

30. Harisankar CNB, Mittal BR, Agrawal KL, Abrar ML, Bhattacharya A. Utility of high fat and low carbohydrate diet in suppressing myocardial FDG uptake. J Nucl Cardiol. 2011;18:926-936.

31. Sadeghi MM, Glover DK, Lanza GM, Fayad ZA, Johnson LL. Imaging atherosclerosis and vulnerable plaque. J Nucl Med. 2010;51(suppl 1):51S-65S. 